\title{
Implementing and designing a secure information system based on the DSSS gold sequence using MATLAB
}

\author{
Kadhim Hassan Kaittan ${ }^{1}$, Samir Jasim Mohammed ${ }^{2}$ \\ ${ }^{1}$ Department of Electronics and Telecommunication, University of Babylon, Babylon, Iraq \\ ${ }^{2}$ Department of Electronics and Telecommunication, Electrical Engineering University of Babylon, Babylon, Iraq
}

\begin{abstract}
Article Info
Article history:

Received Apr 2, 2020

Revised Jan 10, 2021

Accepted Mar 5, 2021

Keywords:

CDMA

DSSS

Gold sequence

$\mathrm{PN}$ sequence

RGB

ABSTRACT

During study of directly spread spectrum systems, noted that this system uses many types of code to spread data through communication process. There are different types of code sequences available with good correlation properties, they often use pesudo sequence for spreading which is to be less efficient in protecting information from the breaches that attack the communications systems from penetration. In this article gold sequence will use, which is consider better and more complex than the rest of the code sequence (pseudo-noise ( $\mathrm{PN})$ sequence, barker code, hadamard code, and others) in terms of the security aspect of the information, thereby it is difficult to detect the information sent in addition to that it is used in code-division multiple access. Design and implement difficult code to protect information in the communication system carried out here by using MATLAB, for sending a binary data and color photo to be sure that this system is working properly with few errors and better information protection than the rest of the other code that is used in direct sequence spread spectrum.
\end{abstract}

This is an open access article under the CC BY-SA license.

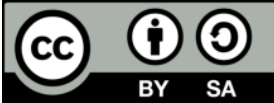

\section{Corresponding Author:}

Kadhim Hassan Kaittan

Department of Electronics and Telecommunication

Electrical engineering, University of Babylon, Babylon, Iraq

Email: gkadim1994@gmail.com

\section{INTRODUCTION}

In each of the systems that need high security such as military and commercial wireless, the information protection system becomes very necessary, because many users rely on wireless networks in general to transfer important information [1]-[4]. This information is vulnerable to attack and thus the reception of information by unwanted receiversFor this purpose, engineers began to study and research in order to obtain information safeguard from hacking against wireless problems. Some of these protections are spread spectrum and encoding technologies. Spread spectrum systems have many advantages, such as: the ability to selectively process information as well as code division multiple access (CDMA) multiple access as well as low-spectrum density, message scanning of eavesdropping, mobility and high resolution range, reject and prevent interference, Initially spread spectrum development, for military purposes (jamming and interference prevention systems) [5]-[7]. Is applied the spectrum technology distributed in most areas of modern communications, for example, wireless networks, cellular phones, as well as global positioning system (GPS) and very small aperture terminal (VSAT) satellite stations.

Spread spectrum techniques are widely used for military and other purposes for commercial purposes. This technique is based on the spreading of the signal on larger frequencies and on a wide range and this is done on the side of the transmitter, then spreading it on the side of the receiver. What we mentioned earlier is in order to obtain the protection of our information in communications systems, due to 
the increasing growth in the number of users, as well as creating a safe environment for our knowledge and privacy in the communications system [8]-[12].

Literature review through the studies previous, which we will look it now, such as Ashush Yonatan et al. [13] this work uses and studies the PN and its difference from other code for DSSS signal and its knowledge of benefits and disadvantages. Sadoudi Said et al. [14] in their study, we propose a new generator of hyperchaos-based deployment symbols for direct-sequence spread spectrum (DSSS) systems. Mohanad Abdulhamid et al. [15] where they studied and designed DSSS of the signal and design s synchronization by Simple code and barely secure protection. Muthna Jasim Fadhil et al. [16] where they used methods to decode and protect information by methods of analysis of neural networks. Oday Kamil Hamid et al. [17] they described their work with a quick approach and used randomness in a matrix-pascal-mathematical way. Rahman Atiqur et al. [18] recommend concepts, features, protection, and internet of things (IoT) certified privacy apps with data protection in our information-driven world.

The problem that most studies suffered from, including previous studies, was the design and implementation of a practical system in our reality that uses randomness to secure private information, building in communications networks, especially after the great development and increasing growth in the development of wireless networks. We solved this problem and secured high private information based on communications networks where We took advantage of a code (gold code) that is common in the communications system and uses its randomness feature, and we have complicated its features to be appropriate to protect our information. The design that we will accomplish in our article and that secures the information will actually cost less than any other costs. It was designed by other studies and from previous studies that we covered.

\section{GOLD SEQUENCE}

Gold sequences known as product codes produced from two m-sequences having the same duration as shown in Figure 1. These two sequences have to be favored pairs of $\mathrm{m}$-sequences. The preferred pairs generated via linearly combining and offsetting positive $\mathrm{m}$-sequences. The correlation residences for gold codes are very proper and have a move correlation value $(-1)$ for many favored m-sequences. The go correlation can be removed by means of attaching ' 0 ' to the gold sequences. Actually, two $\mathrm{M}$ code sequences with 0 move-correlation among them may be generated through padding 0 to the original gold sequences. These sequences are known as orthogonal gold sequences [19]-[21].

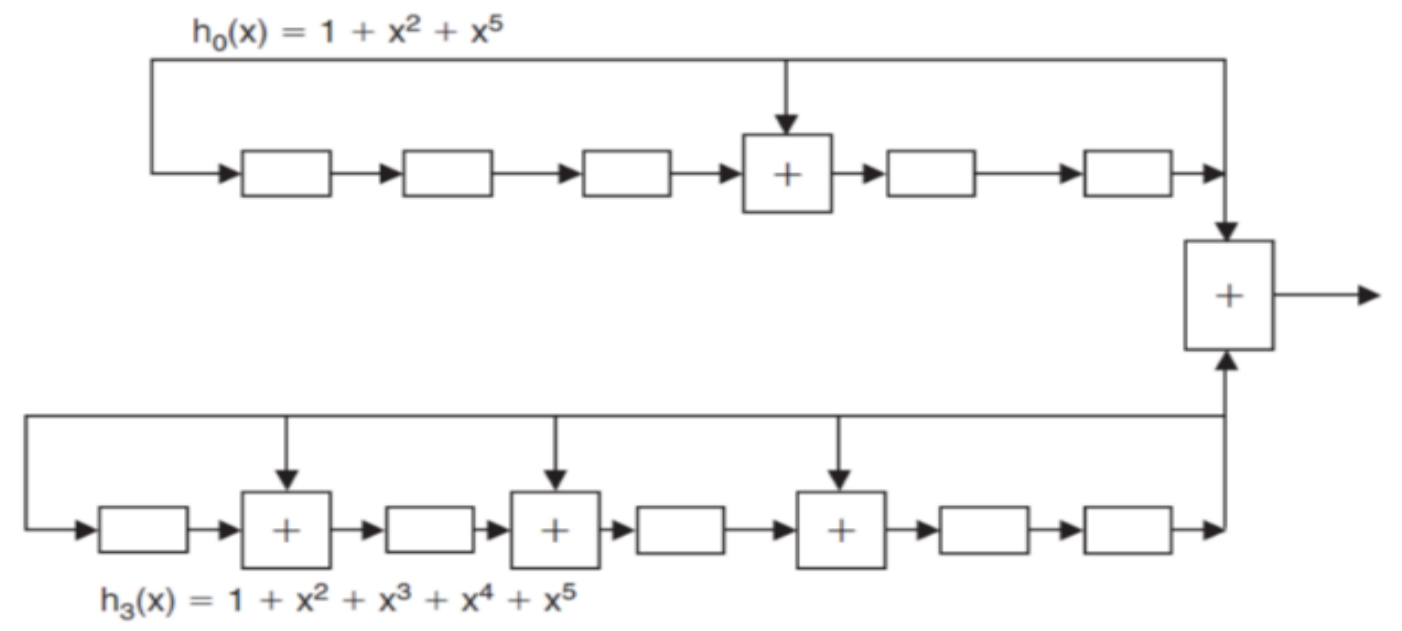

Figure 1. Gold generator block diagram

Gold codes have worst auto-correlationproperties but they have better cross-correlation properties. Where it is of great importance in terms of complexity in the length of the code it is also difficult to guess the code from the hackers. Cross-correlation properties shown in the Figure 2. Where this code differs from the rest of the other code in this regard, and this distinguishes it in the protection of information [22], [23], as well as in terms of complexity and in the transmission of one of the most secure signals in the communication system. 


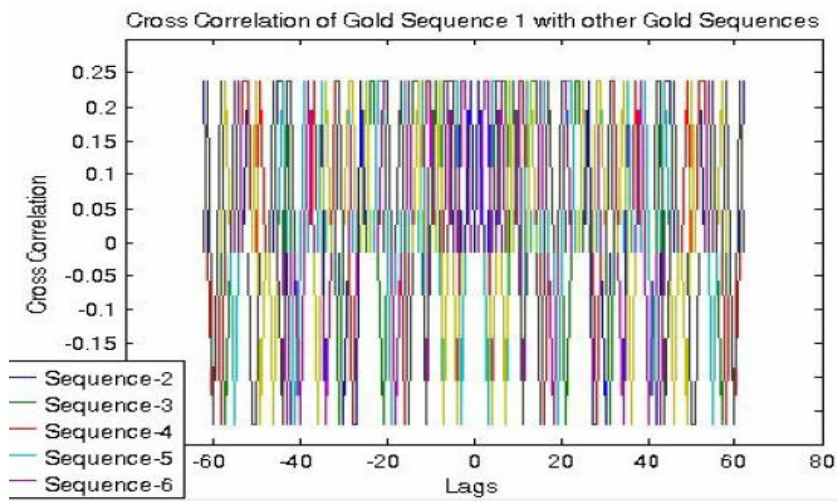

Figure 2. Cross-correlation properties

\section{RECEIVER PERFORMANCE IN THE PRESENCE OF INTERFERENCE}

One of the major properties of all spread spectrum systems is their ability to reject interference. In a properly synchronized receiver, DS signal is despreaded by multiplying it (i.e. correlation) with a locally generated PN sequence replica [24]-[27]. On the other hand, a jammer with an incident power equal to $\mathrm{J}$ and a bandwidth of $\mathrm{BJ}$ is spreaded so that the jammer power spectral density (SJ) at the correlator output is equal to as in (2).

$$
S_{J}=\frac{J}{\left(B_{J}+B_{S S}\right)}
$$

The above expression clearly shows that a relatively narrow band jammer is the most effective interference to a direct sequence receiver, since it is typically able to place a great fraction of incident jamming power in the baseband of the victim receiver during communication, then is a relatively wider band jammer [28].

\section{DESIGN AND IMPLEMENT THE FEATURES OF THIS SYSTEM}

In our study, we will discuss our design and implementation of a secure communications system in data protection and high speed data transfer with few error rates where it will be sent binary data and color image red/green/blue (RGB) using MATLAB/SIMULINK.

\subsection{Using gold sequences with BPSK and QPSK of binary data}

The gold sequence generator block generates a gold sequence. Gold sequences form a large class of sequences that have good periodic cross correlation properties. In Figure 3 illustrates the block diagram of the general DS/SS with gold sequence of binary phase shift keying (BPSK) and quadrature phase shift keying (QPSK) modulation. In the implementation of this system the parameters will be is being as: 1) Preferred polynomial (1) of the code sequence is $1+x+x^{6} ; 2$ ) Preferred polynomial (2) of the code sequence is $1+x+x^{2}+x^{5}+x^{6}$; 3) Data rate of signal transmitted $1000 \mathrm{~Hz}$; 4) Code rate of gold sequence $32 \mathrm{KHz} ; 5$ ) Output data type is double. In this system, it was done direct sequence spread spectrum of Random integer generator via gold sequence then the signal was modulation by BPSK and QPSK. Then the signal was sent over additive white gaussian noise (AWGN) channel. Where it was changed $E b / N 0$ to know the percentage of error in the sent messages. The processing gain of the system $15.05 \mathrm{~dB}$.

\subsection{Using gold sequences with change modulation of image (RGB)}

In this case we have changed the sender from Binary data to image to demonstrate that the system can be built in practice and that it is an advanced system that sends images and other signals. Figure 4 illustrate the block diagram of the general direct sequence/spread spectrum (DS/SS) with gold sequence of BPSK (Log-likelihood ratio) transmitter and decision the parameters of this system are as in the following points: 1) Dimensions of the submitted RGB image $(960 * 639)$ array pixels. In addition, number of byte equal (613440-byte). And number of bit equal (4907520 bit) sample time of image-transmitted equal $(490.752 \mathrm{sec})$ and Sample time of bit transmitted Tb equal $(0.1 \mathrm{~ms}) ; 2)$ Sample time of code sequence Tc equal $(0.01 \mathrm{~ms}) ; 3)$

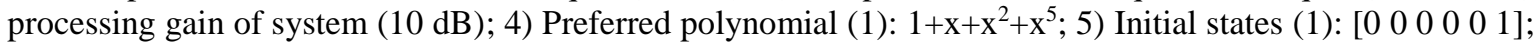
5) Preferred polynomial (2): $1+x^{2}+x^{3}+x^{5}$; 6) Initial states (2): [ $\left.10 \begin{array}{lllll}0 & 0 & 0 & 0 & 1\end{array}\right]$; 7) Output data type: binary. 


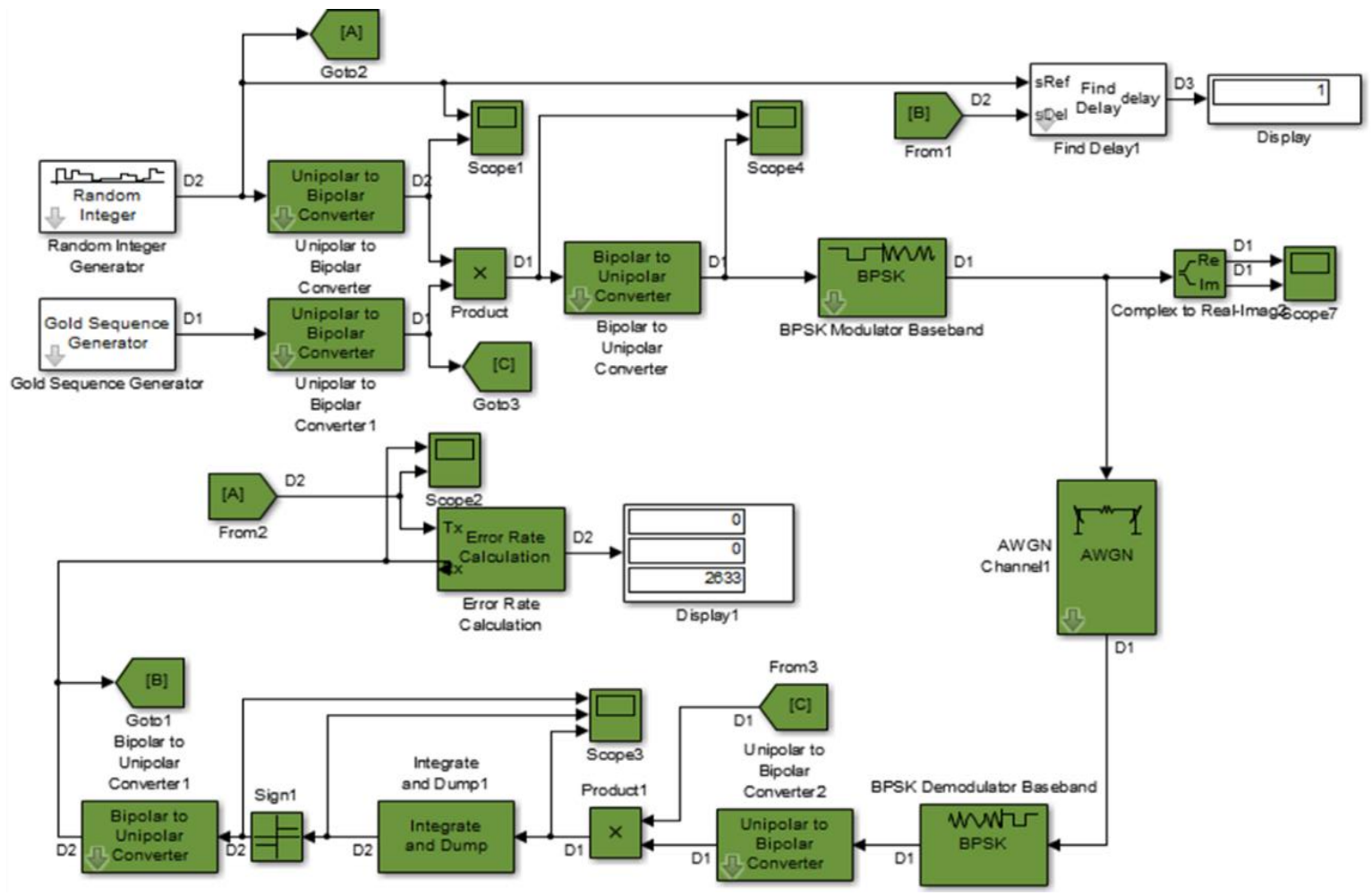

Figure 3. Simulink block diagram of DS/SS system of gold sequence with change modulation

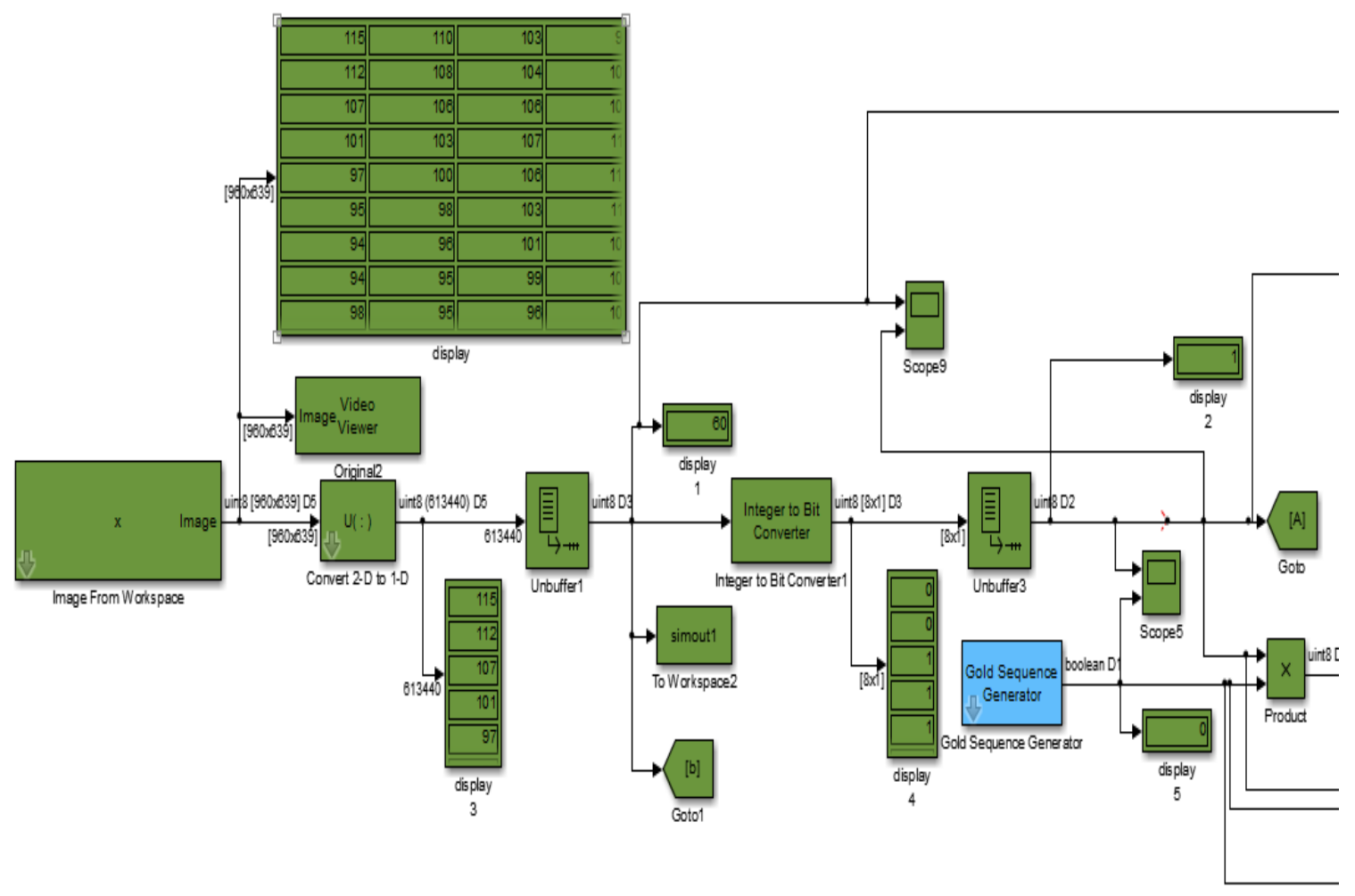

(a)

Figure 4. Simulink block diagram of DS/SS system of gold sequence of RGB image with change modulation, (a) Transmitter system 


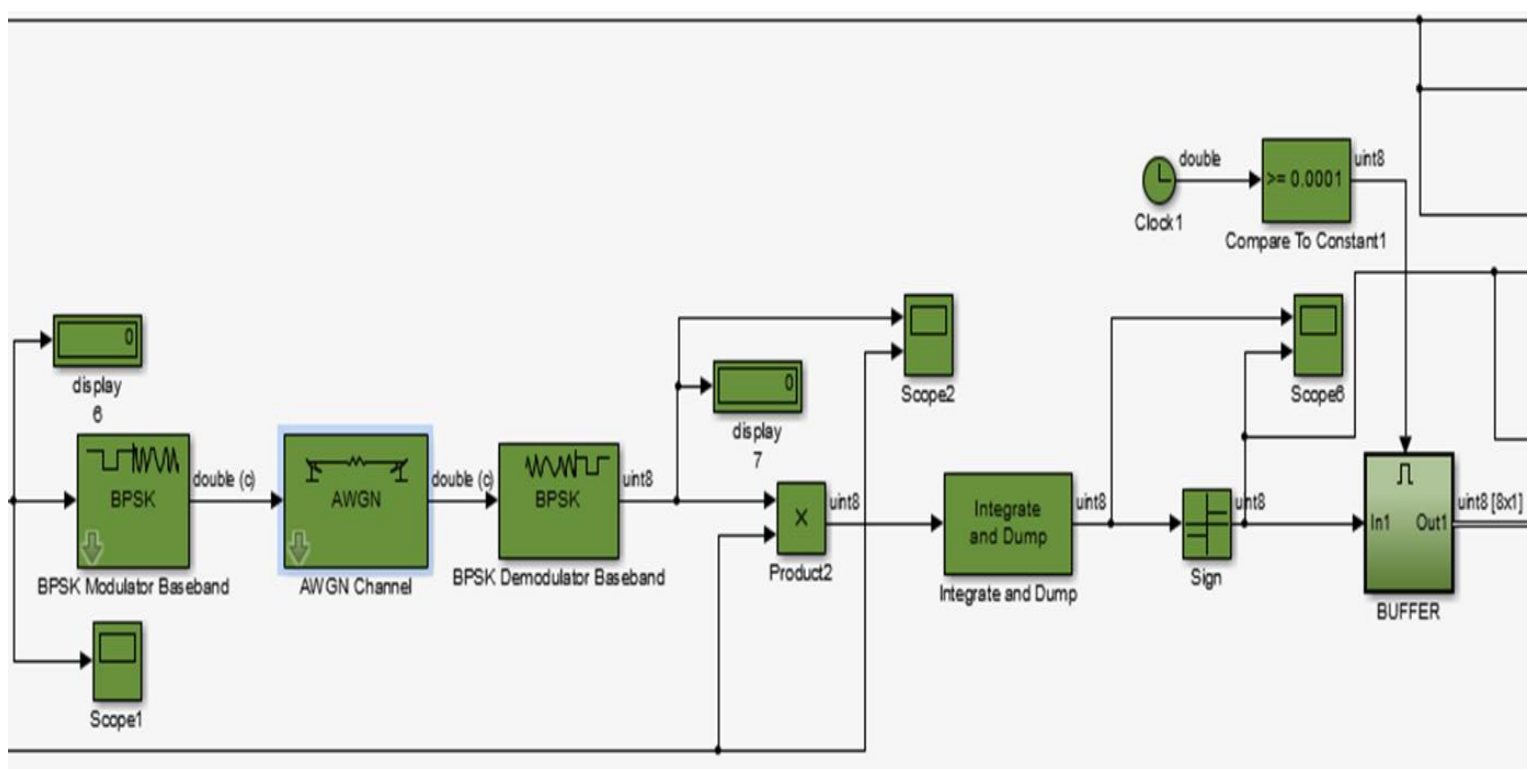

(b)

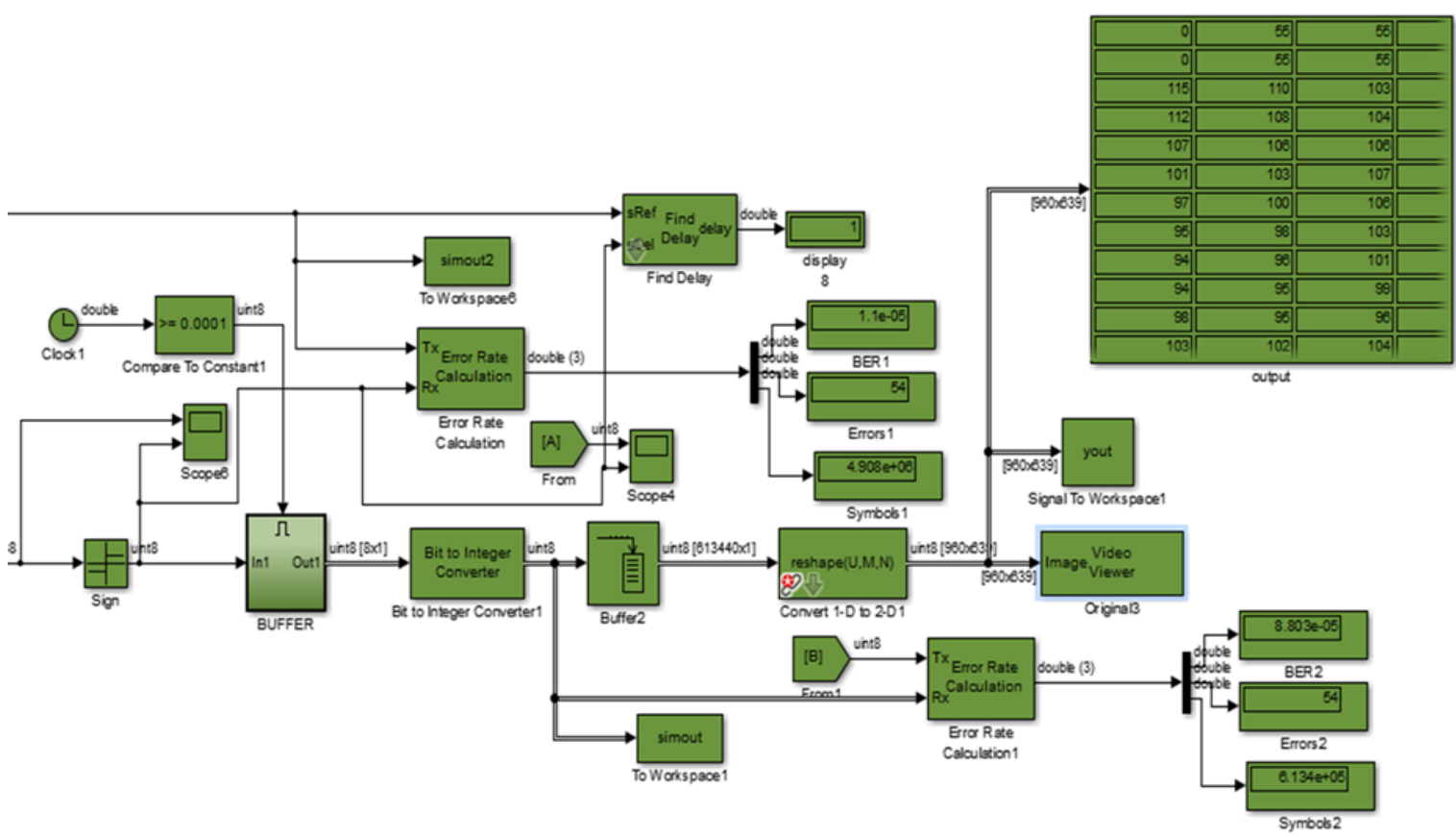

(c)

Figure 4. Simulink block diagram of DS/SS system of gold sequence of RGB image with change modulation,

(b) Channel, (c) Receiver system (continue)

\section{RESULTS AND DISCUSSION}

In these results, we will look at the details of sending and receiving in the communication system that we designed and implemented so that these results differ from the results of previous studies from the practical side of the system since the previous studies used types of code in which the signals are vulnerable to penetration and attacks from inside and outside the communication system in addition to that. The previous studies have sent signals, but we have sent the signals using the most complex and longest code in the communication system to protect the information sent in the communication system in addition to the fact hat these signals that we have sent are signals that exist in reality work such as picture. In our system, we sent data and image in a few errors percentage and this design was not fulfilled by previous studies as was previously explained. 


\subsection{DSSS of gold sequence when sent binary data}

After we explained previously the system-specific parameters and the signal was sent random binary data where he was time of transmitter of the signal $1 \mathrm{~ms}$ and data rate of the signal $1 \mathrm{kbit} / \mathrm{s}$ after we hit the signal by gold code that's where the value of chip rate of the code is $32 \mathrm{kbit} / \mathrm{s}$. And we got a secure signal that was able to be sent in the communication system without attacks by any party. As the figures that we got in this system were as below, and we will look at them in detail.

Where the Figure 5(a) represents transmitter, signal was this reference frequency high and of high signal to noise ratio and Figure 5(b) is the spreading signal after spread of the transmitter signal in order to get a secure signal in the communiciation system where the processing gain equal $15.05 \mathrm{~dB}$. Then sent through channel AWGN in this channel we change SNR (-15dB to $15 \mathrm{~dB})$ of the signal and add noise to siganal in order to calculate the bit error rate (BER) where was the result of the probability of error equal $(0.000105,0.00020,0.0030 \ldots .$.$) where the probability of error increases when the reduce signal to noise ratio$ (SNR) to minus dB conversely. Figure 5(c) represent received signal. In comparison with previous studies, as some of the researchers we mentioned used codes that were not complicated by randomness, such as the code that we implemented, and others used different methods to secure the signals, and their results were close to our results, but we cost less and high data rate of data.

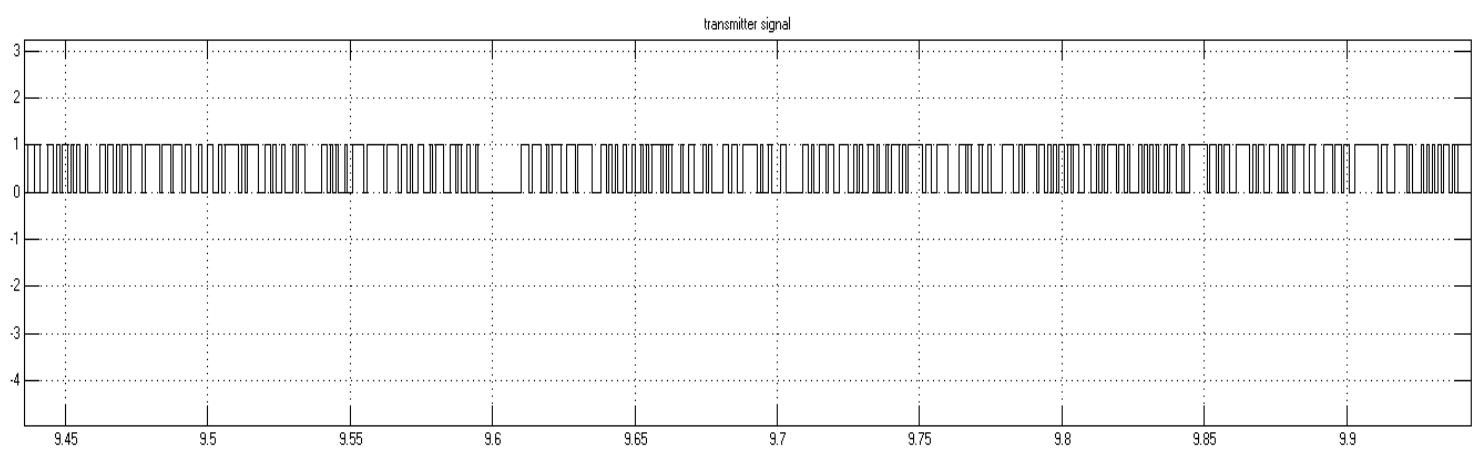

(a)

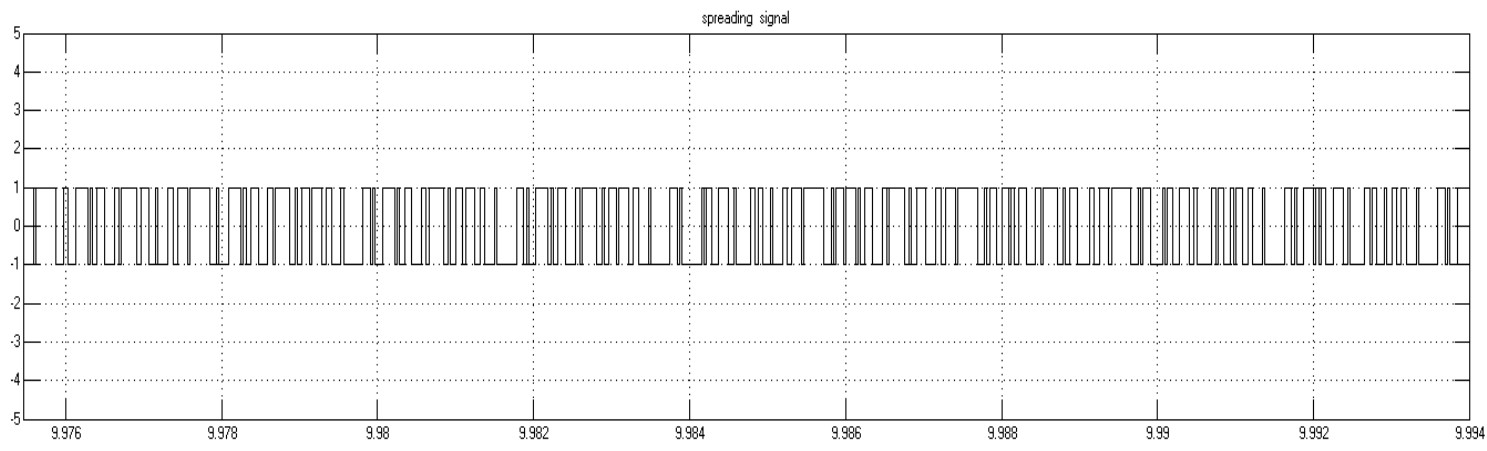

(b)

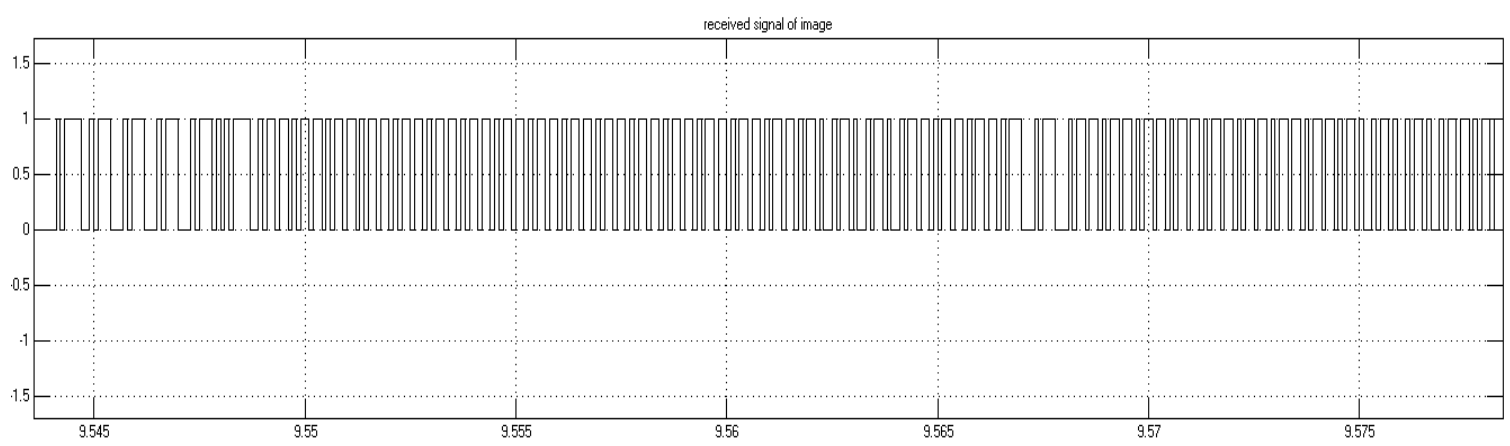

(c)

Figure 5. MATLAB/SIMULINK, (a) Transmitter signal, (b) Spreading signal, (c) Received signal 


\subsection{DSSS of gold sequence when sent image (RGB) data}

In this case has been sent image $(960 * 639)$ RGB during the AWGN channel after the multipier by gold code. Terms of the parameters system of transmitter and receiving mentioned previously forms and below are the results of the system. In the results that appeared to us after we designed and implemented, we calculated a few errors where was the number of errors $(24 \mathrm{bit})$ this number is small in relation to the size of the image, the number of decisions taken (4907520 bit) In addition to,the probability bit error equal its approach to almost zero Despite the large amount of information that had been sent, and this previous studies could not verify it. In Figure 6(a) represent transmitter signal of image and Figure 6(b) represent spreading signal of image and Figure 6(c) represent recived signal after decision circuit in the receive. Compared to previous studies, they did not address their studies to send and secure high protection of information such as photos (RGB) and videos, but we touched upon this and using strong randomness against intentional and unintentional attacks and attacks. All of the above mentioned as direct signals in the wireless communication system, and we will look at the picture sent and received in Figure 7. Whereas Figure 7 it is divided into two parts figure. Figure 7(a) photo (RGB) or image transmitter and Figure 7(b) photo (RGB) or image receiver. After it has been filled out and sent through channel pick it up and hit it with the random code again to get it. And Figure 8 the explains his results and the difference between using different types of code and different modulation to protect digital information for communications.

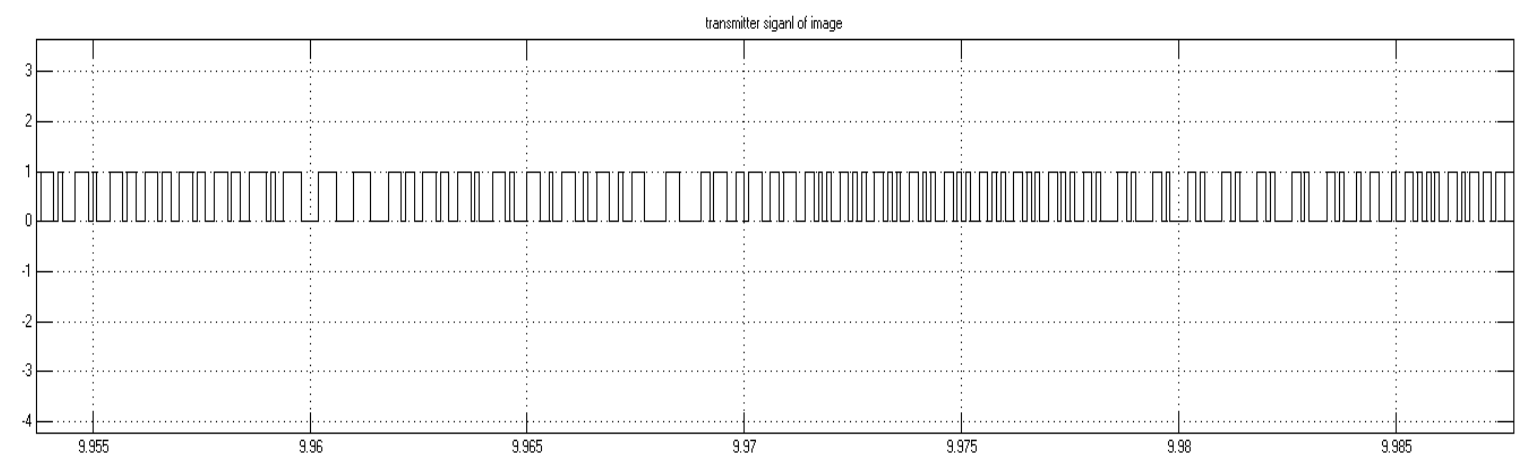

(a)

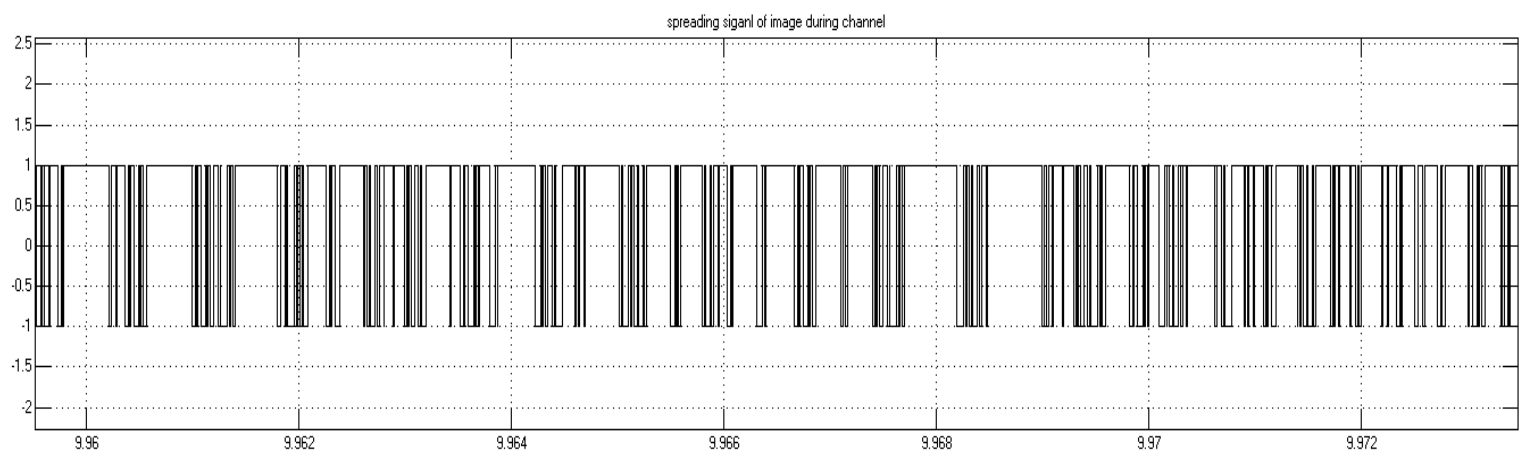

(b)

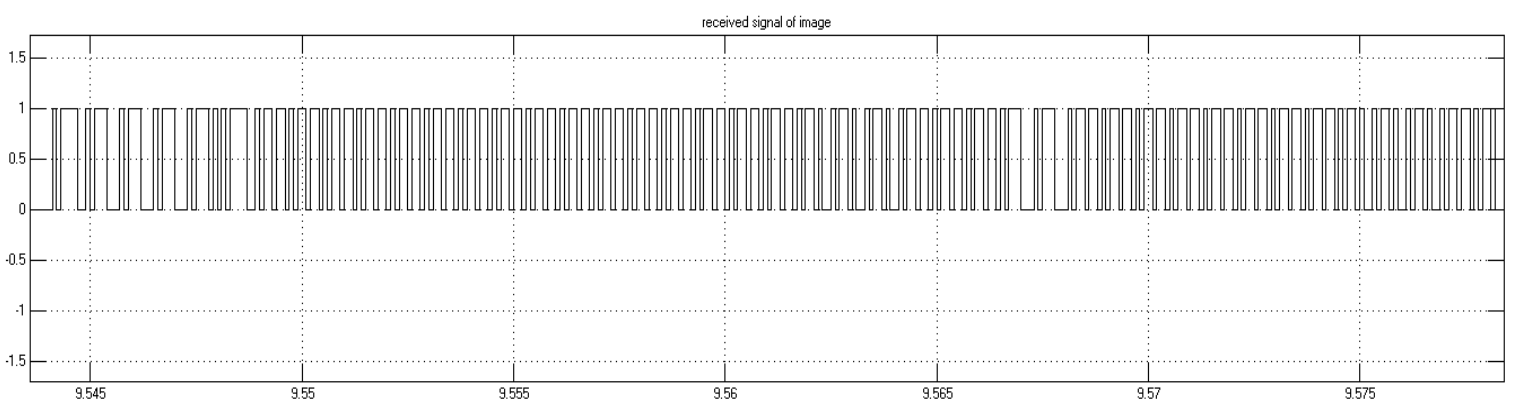

(c)

Figure 6. Matlab/SIMULINK, (a) Transmitter signal, (b) Spreading signal during channel, (c) Received signal 


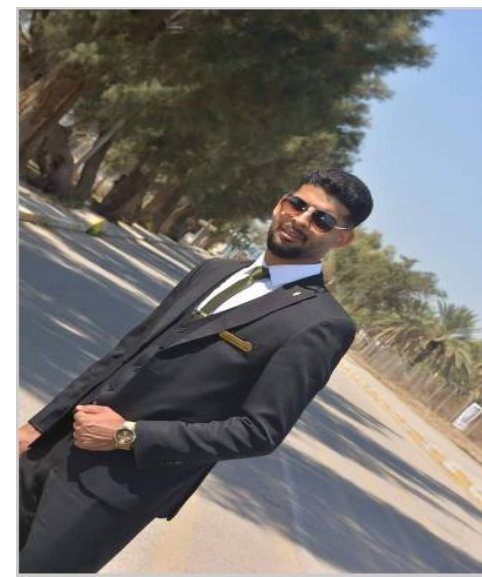

(a)

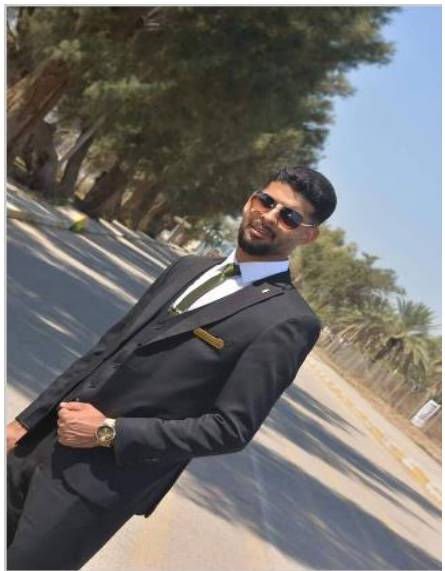

(b)

Figure 7. These figures are, (a) Transmitter photo, (b) Receiver photo

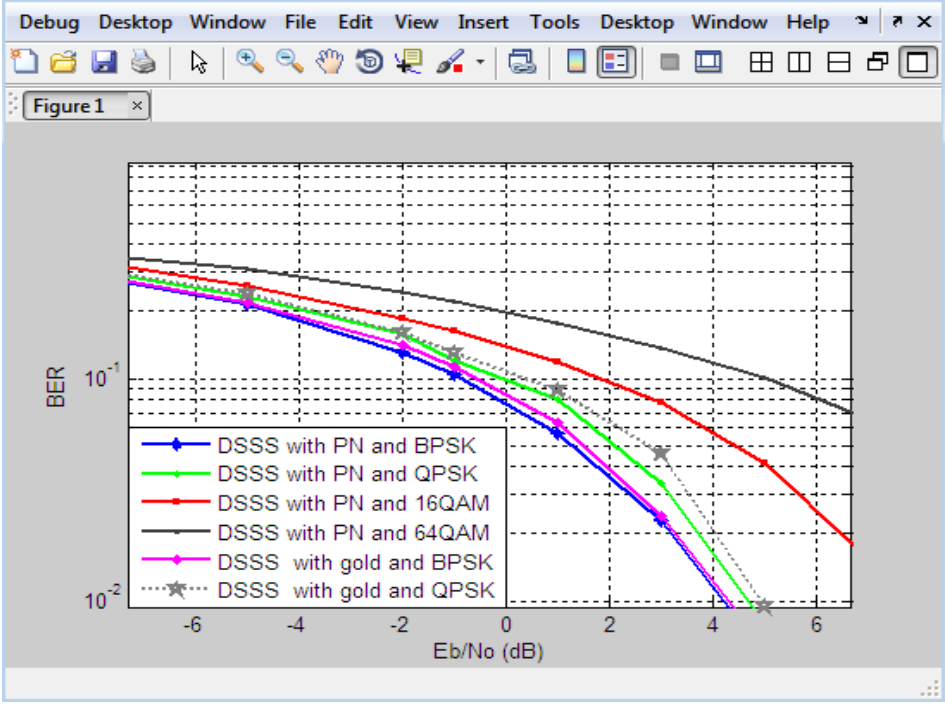

Figure 8. DSSS with different code and modulation

\section{CONCLUSION}

Design and implementation achieved in this articles, used a random code to secure the information required to send over wireless communication networks. The results gets from sent pictures was good with miner mistakes, in addition the power of the transmitted reduced which is consider an advantage of randomization reduces the transmission power at the expense of the transmission bandwidth and future scope use orthogonal variable spreading factor (OVSF) for spread code and the study system under change channel and modulation.

\section{REFERENCES}

[1] U. Narayanan, V. Paul, and S. Joseph, "A light weight encryption over big data in information stockpiling on cloud," Indonesian Journal of Electrical Engineering and Computer Science (IJEECS), vol. 17, no. 1, pp. 389-397, 2020, doi: 10.11591/ijeecs.v17.i1.pp389-397.

[2] Ambika, V. Kaba, "Implementation of DSSS System using Chaotic Sequence using MATLAB and VHDL," International Journal of Computer Science and Mobile Computing, vol. 4, no. 5, pp. 598-607, 2015.

[3] M. Viswanathan, "Simulation of Digital Communication Systems Using Matlab," Mathuranathan Viswanathan at Smashwords, 2013. 
[4] M. B. Mollah and M. R. Islam, "Comparative analysis of Gold Codes with PN codes using correlation property in CDMA technology," 2012 International Conference on Computer Communication and Informatics, Coimbatore, India, 2012, pp. 1-6, doi: 10.1109/ICCCI.2012.6158894.

[5] J. K. Kim, M. Lee, K. I. Lee, K. W. Park, and K. S. Woo, "MATLAB/Simulink for Digital Communication," Hongrung Publishing Comany, 2013.

[6] H. Singh, E. R. Singh, and N. Kaur, "Implementing Hierarchical Clustering Method for Multiple Sequence Alignment and Phylogenetic Tree Construction," International Journal of Computer Science, Engineering and Information Technology (IJCSEIT), 2013, doi: 10.5121/ijcseit.2013.3101.

[7] A. S. M. Anuar, W. N. W. Muhamad, D. M. Ali, S. S. Sarnin, and N. A. Wahab, "A review on link adaptation techniques for energy efficiency and QoS in IEEE802.11 WLAN," Indonesian Journal of Electrical Engineering and Computer Science, vol. 17, no. 2, pp. 988-996, 2019, doi: 10.11591/ijeecs.v17.i2.pp988-996.

[8] C. C. -. Chen, K. Yao and E. Biglieri, "Optimal spread spectrum sequences-constructed from Gold codes," Globecom '00 - IEEE. Global Telecommunications Conference. Conference Record (Cat. No.00CH37137), San Francisco, CA, USA, 2000, pp. 867-871 vol. 2, doi: 10.1109/GLOCOM.2000.891262.

[9] R. Poisel, "Modern Communications Jamming: Principles and Techniques," Artech House, 2011.

[10] E. Gopi, “Multi-Disciplinary Digital Signal Processing," A Functional Approach Using Matlab. Suiza: Springer, 2018.

[11] N. H. Ja'afar and A. Ahmad, "Algorithm development and hardware implementation for medical image compression system: A review," Indonesian Journal of Electrical Engineering and Computer Science (IJEECS), vol. 18, no. 3, pp. 1331-1341, 2020, doi: 10.11591/ijeecs.v18.i3.pp1331-1341.

[12] A. Tikhomirov, E. Omelvanchuk, A. Semenova, A. Smirnov and A. Bakhtin, "Direct Sequence Spread Spectrum System Noise and Interference Immunity Analysis," 2018 IEEE East-West Design \& Test Symposium (EWDTS), Kazan, Russia, 2018, pp. 1-6, doi: 10.1109/EWDTS.2018.8524661.

[13] Y. Ashush and S. Engelberg, "Generation of "Optimal" PN Sequences for Use in Direct Sequence Spread Spectrum," 2018 IEEE International Conference on the Science of Electrical Engineering in Israel (ICSEE), Eilat, Israel, 2018, pp. 1-4, doi: 10.1109/ICSEE.2018.8646246.

[14] S. Sadoudi, I. Fennouh, and C. Tanougast, "Hyperchaos-based spreading codes generator for DS-CDMA communication systems," Journal of Circuits, Systems and Computers, vol. 27, no. 13, pp. 1-23, 2018, doi: $10.1142 / \mathrm{S} 0218126618502079$.

[15] M. Abdulhamid and H. Omar, "Code Tracking of DSSS Signal over AWGN Channel," The Scientific Bulletin of Electrical Engineering Faculty, vol. 19, no. 1, pp. 13-17, 2019, doi: 10.1515/sbeef-2019-0003.

[16] M. J. Fadhil, M. A. Naji, and G. A. Salman, "Transceiver error reduction by design prototype system based on neural network analysis method," Indonesian Journal of Electrical Engineering and Computer Science (IJEECS), vol. 18, no. 3, pp. 1244-1251, 2020, doi: 10.11591/ijeecs.v18.i3.pp1244-1251.

[17] O. K. Hamid, R. B. Abduljabbar, and N. J. Alhyani, "Fast and robust approach for data security in communication channel using pascal matrix," Indonesian Journal of Electrical Engineering and Computer Science (IJEECS), vol. 19, no. 1, pp. 248-256, 2020, doi: 10.11591/ijeecs.v19.i1.pp248-256.

[18] A. Rahman, "Mobile edge computing for internet of things (IoT): Security and privacy issues," Indonesian Journal of Electrical Engineering and Computer Science (IJEECS), vol. 18, no. 3, p. 1486, 2020, doi: 10.11591/ijeecs.v18.i3.pp1486-1493.

[19] L. Hanzo, M. Münster, B. J. Choi, and T. Keller, "OFDM and MC-CDMA for Broadband Multi-User Communications,'” WLANs and Broadcasting, 2005.

[20] H. Mane, K. Y. K. Kumari, G. B. N. Vamsidhar, A. Kartheek, A. V. Kumar, and M. V. Rao, "Direct Sequence Spread Spectrum transmission and reception using compression techniques," 2015 International Conference on Smart Technologies and Management for Computing, Communication, Controls, Energy and Materials (ICSTM), Avadi, India, 2015, pp. 344-347, doi: 10.1109/ICSTM.2015.7225439.

[21] S.S.S. Sugi and C. V. Joe, "Spread Spectrum Modulation Techniques using MATLAB," International Journal of Innovative Research In Technology, vol. 2, no. 3, pp. 47-52, August, 2015.

[22] A. Tikhomirov, E. Omelvanchuk, A. Semenova, A. Smirnov and A. Bakhtin, "Direct Sequence Spread Spectrum System Noise and Interference Immunity Analysis," 2018 IEEE East-West Design \& Test Symposium (EWDTS), Kazan, Russia, 2018, pp. 1-6, doi: 10.1109/EWDTS.2018.8524661.

[23] Y. Wei, S. Fang, X. Wang, and S. Huang, "Blind estimation of the PN sequence of a DSSS signal using a modified online unsupervised learning machine," Sensors, vol. 19, no. 2, 2019, doi: 10.3390/s19020354.

[24] S. Lei and Z. Zhijing, "Blind Estimation of Pseudo-random Sequences of the Carrier Modulated Direct Sequence Spread Spectrum Signals in Asynchronous Uplink System Using Space-Time Complex-ICA," 2009 Pacific-Asia Conference on Circuits, Communications and Systems, Chengdu, China, 2009, pp. 526-530, doi: 10.1109/PACCS.2009.74.

[25] A. Kumar and M. Gupta, "Design , comparative study and analysis of CDMA for different modulation techniques," Egyptian Informatics Journal, pp. 351-365, 2015, doi: 10.1016/j.eij.2015.07.004.

[26] M. B. Pursley, "Direct-sequence spread-spectrum communications for multipath channels," in IEEE Transactions on Microwave Theory and Techniques, vol. 50, no. 3, pp. 653-661, March 2002, doi: 10.1109/22.989950.

[27] M. R. Karim, “W-CDMA and cdma2000 for 3G Mobile Networks,” MR Karim and Lucent Technologies, Inc, 2002.

[28] Hikmat Najem Al-Shamary, "Design and Implementation of Direct Sequence Spread Spectrum System Design and Implementation of Direct Sequence Spread Spectrum System Using Field Programmable Gate Array,” Ph.D. dissertation, Department of Electrical Engineering, University of Technology, June 2004. 